14,15

\title{
Полевые транзисторы с высокой подвижностью и малым гистерезисом передаточных характеристик на основе пленок $\mathrm{CH}_{3} \mathrm{NH}_{3} \mathrm{PbBr}_{3}$
}

\author{
(C) А.Н. Алешин, И.П. Щербаков, И.Н. Трапезникова, В.Н. Петров
}

Физико-технический институт им. А.Ф. Иофрфе РАН,

Санкт-Петербург, Россия

E-mail: aleshin@transport.ioffe.ru

(Поступила в Редакцию 3 апреля 2017 г.)

Получены полевые транзисторные (ПТ) структуры на основе растворимых металлорганических перовскитов - $\mathrm{CH}_{3} \mathrm{NH}_{3} \mathrm{PbBr}_{3}$ и исследованы их электрические свойства. Показано, что ПТ на основе пленок $\mathrm{CH}_{3} \mathrm{NH}_{3} \mathrm{PbBr}_{3}$ демонстрируют вольт-амперные характеристики (BAX), характерные для амбиполярных ПТ с режимом насыщения. Обнаружено, что передаточные характеристики ПТ на основе $\mathrm{CH}_{3} \mathrm{NH}_{3} \mathrm{PbBr}_{3}$ обладают незначительным гистерезисом и слабо зависят от напряжения на стоке-истоке. Значения подвижности носителей заряда (дырок), рассчитанные из ВАХ ПТ на основе $\mathrm{CH}_{3} \mathrm{NH}_{3} \mathrm{PbBr}_{3}$ при $300 \mathrm{~K}$ в режимах насыщения и слабых полей, составили $\sim 5 \mathrm{~cm}^{2} / \mathrm{Vs}$ и $\sim 2 \mathrm{~cm}^{2} / \mathrm{Vs}$ соответственно, а подвижность электронов $\sim 3 \mathrm{~cm}^{2} / \mathrm{Vs}$, что превышает значение подвижности $\sim 1 \mathrm{~cm}^{2} / \mathrm{Vs}$, полученное ранее для ПТ на основе $\mathrm{CH}_{3} \mathrm{NH}_{3} \mathrm{PbI}_{3}$.

Работа выполнена при частичной поддержке Программ фундаментальных исследований ПРАН $1.8 П$ и 1.25П и гранта РФФИ № 15-02-01897.

DOI: 10.21883/FTT.2017.12.45248.108

\section{1. Введение}

Материалы на основе металлорганических перовскитов в настоящее время привлекают очень большое внимание в связи с перспективами их использования в качестве активных слоев высокоэффективных солнечных элементов (СЭ) [1,2]. В 2016 г. КПД СЭ на основе металлорганических перовскитов $\mathrm{CH}_{3} \mathrm{NH}_{3} M X_{3}$ $(M=\mathrm{Pb}, \mathrm{Sn}, X=\mathrm{Cl}, \mathrm{Br}, \mathrm{I})$ достиг $\sim 22.1 \% \quad[1,3,4]$. Такой высокий КПД перовскитовых СЭ стал возможен благодаря эффективному поглощению света в видимом спектральном диапазоне, сбалансированным транспортным свойствам и большой диффузионной длине носителей заряда в таких материалах [5]. Эти свойства делают материалы на основе металлорганических перовскитов перспективными для применения в качестве активных полупроводниковых слоев в полевых транзисторах (ПТ). В последние годы были получены и исследованы свойства ПТ на основе пленок различных металлорганических перовскитов. Так, в ПТ на основе пленок $\mathrm{CH}_{3} \mathrm{NH}_{3} \mathrm{PbI}_{3}$, нанесенных центрифугированием, были получены значения полевой подвижности дырок $\left(\mu_{\text {hole }}\right)$ при $300 \mathrm{~K} \mu_{\text {hole }} \sim 10^{-5} \mathrm{~cm}^{2} / \mathrm{Vs}[6]$ и $\mu_{\text {hole }} \sim 2.1 \cdot 10^{-2} \mathrm{~cm}^{2} / \mathrm{Vs}[7]$, а значения подвижности электронов $\left(\mu_{\text {elec }}\right) \sim 10^{-2} \mathrm{~cm}^{2} / \mathrm{Vs}[7,8]$, тогда как в ПТ на основе микрокристаллов $\mathrm{CH}_{3} \mathrm{NH}_{3} \mathrm{PbI}_{3}$ значения подвижности составили $\mu_{\text {hole }} \sim 1-2.5 \mathrm{~cm}^{2} / \mathrm{Vs}$ [9]. В ПТ на основе $\mathrm{CH}_{3} \mathrm{NH}_{3} \mathrm{PbI}_{3-x} \mathrm{Cl}_{x}$ был продемонстрирован амбиполярный режим работы при $300 \mathrm{~K}$ со сбалансированными значениями $\mu_{\text {hole }}$ и $\mu_{\text {elec }}(\sim 1.3$ и $\sim 1.0 \mathrm{~cm}^{2} / \mathrm{Vs}$ соответственно) [5]. С другой стороны, значения $\mu_{\text {hole }}(300) \sim 0.6,2.6$, и $\sim 0.78 \mathrm{~cm}^{2} / \mathrm{Vs}$ были получены для 2D-пленок металлорганических перовскитов $\left(\mathrm{C}_{6} \mathrm{H}_{5} \mathrm{C}_{2} \mathrm{H}_{4} \mathrm{NH}_{3}\right)_{2} \mathrm{SnI}_{4}$, нанесенных на подложки методом центрифугирования [10] и напылением в вакууме [11] соответственно. Недавно рекордные значения $\mu_{\text {hole }} \sim 15 \mathrm{~cm}^{2} / \mathrm{Vs}$ при $300 \mathrm{~K}$ с незначительным гистерезисом вольт-амперных характеристик (BAX) были получены в ПТ на основе $\left(\mathrm{C}_{6} \mathrm{H}_{5} \mathrm{C}_{2} \mathrm{H}_{4} \mathrm{NH}_{3}\right)_{2} \mathrm{SnI}_{4}$ при предварительном нанесении на подложки монослоя самоорганизующихся молекул, содержащих концевые группы иодида аммония ( $\left.\mathrm{NH}_{3} \mathrm{I}-\mathrm{SAM}\right)$ [12]. Возможными источниками относительно низких значений $\mu_{\text {hole }}$ и $\mu_{\text {elec }}$ наблюдаемых в перовскитовых ПТ, являются невысокое качество пленок металлорганических перовскитов, высокая плотность ловушек и неэффективная инжекция носителей заряда. Наблюдавшийся в большинстве работ большой гистерезис выходных и передаточных характеристик, детально исследованный в работе [13] для ПТ на основе $\mathrm{CH}_{3} \mathrm{NH}_{3} \mathrm{PbI}_{3}$ с максимальной $\mu_{\text {hole }} \sim 0.5 \mathrm{~cm}^{2} / \mathrm{Vs}$, является еще одной серьезной проблемой для повышения подвижности перовскитовых ПТ. Таким образом, природа транспорта в ПТ на основе металлорганических перовскитов остается исследованной недостаточно, а вопросы, связанные с повышением подвижности и уменьшением гистерезиса BAX в таких ПТ, в настоящее время являются актуальными и открытыми для обсуждения.

Нами получены ПТ на основе пленок растворимых металлорганических перовскитов - $\mathrm{CH}_{3} \mathrm{NH}_{3} \mathrm{PbBr}_{3}$ и исследованы их электрические и оптические свойства. Полученные ПТ структуры демонстрируют ВАХ, характерные для амбиполярных ПТ с режимом насыщения. Пе- 
редаточные характеристики ПТ на основе $\mathrm{CH}_{3} \mathrm{NH}_{3} \mathrm{PbBr}_{3}$ обладают незначительным гистерезисом и слабо зависят от напряжения на стоке-истоке. Значения подвижности носителей заряда (дырок) при $300 \mathrm{~K}$, рассчитанные из BAX ПТ на основе $\mathrm{CH}_{3} \mathrm{NH}_{3} \mathrm{PbBr}_{3}$ в режимах насыщения и слабых полей, составляют $\sim 5 \mathrm{~cm}^{2} / \mathrm{Vs}$ и $\sim 2 \mathrm{~cm}^{2} / \mathrm{Vs}$, а подвижность электронов $\sim 3 \mathrm{~cm}^{2} / \mathrm{Vs}$.

\section{2. Объекты и методы исследования}

Для приготовления образцов были использованы порошки металлорганических перовскитов $\mathrm{CH}_{3} \mathrm{NH}_{3} \mathrm{PbBr}_{3}$ с шириной запрещенной зоны $E_{g} \sim 2.3 \mathrm{eV}$, приобретенные у кампании Xi'an Polymer Light Technology Corp. и использованные без дополнительной обработки. На рис. 1, a показана химическая структура металлорганического перовскита $-\mathrm{CH}_{3} \mathrm{NH}_{3} \mathrm{PbBr}_{3}$ (обозначается также как $\left.\mathrm{MAPbBr}_{3}\right)$ [4], использованного в нашей работе. Были изготовлены ПТ-структуры на основе $\mathrm{CH}_{3} \mathrm{NH}_{3} \mathrm{PbBr}_{3}$, включающие в себя $n-\mathrm{Si} / \mathrm{SiO}_{2} / \mathrm{Au} / \mathrm{CH}_{3} \mathrm{NH}_{3} \mathrm{PbBr}_{3} / \mathrm{Al}$ (рис. 1,b). В качестве затвора использовалась подложка из сильно легированного $\mathrm{n}+$ кремния с термически выращенным слоем $\mathrm{SiO}_{2}$ толщиной $200 \mathrm{~nm}$ в качестве диэлектрика с нанесенными методом термического испарения золотыми (Au) и алюминиевыми (Al) электродами. Расстояние между $\mathrm{Au}-\mathrm{Al}$ и $\mathrm{Au}-\mathrm{Au}$ электродами составляло $\sim 7 \mu \mathrm{m}$ и $\sim 15 \mu \mathrm{m}$ соответственно, ширина электродов $\sim 1 \mathrm{~mm}$. Порошок $\mathrm{CH}_{3} \mathrm{NH}_{3} \mathrm{PbBr}_{3}$ растворялся в DMA - $N$, $N$-Dimethylacetamide, затем раствор перемешивался ультразвуком в течение 10 min на ультразвуковой мешалке Bandelin Sonopuls HD $2070(f \sim 20 \mathrm{kHz})$. Полученный раствор наносился на $\mathrm{Si} / \mathrm{SiO}_{2}$ подложки с $\mathrm{Au}$ и $\mathrm{Al}$ электродами методами полива или центрифугирования (при $3000 \mathrm{rpm}$ ). Нанесенные таким образом пленки высушивались при $100^{\circ} \mathrm{C}$ в атмосфере $\mathrm{N}_{2}$ в течение $15 \mathrm{~min}$. Толщина пленок, полученных методом полива, составила $\sim 0.6 \mu \mathrm{m}$ согласно результатам атомно-силовой микроскопии (ACM). Спектры поглощения пленок металлорганических перовскитов, нанесенных на кварцевые подложки (толщина $\sim 1 \mu \mathrm{m})$, исследовались с помощью спектрометра Cary-50 (Varian). Морфология пленок $\mathrm{CH}_{3} \mathrm{NH}_{3} \mathrm{PbBr}_{3}$ исследовалась с помощью $\mathrm{ACM}-\mathrm{P} 47-$ Solver NT-MDT. Спектры фотолюминесценции (ФЛ) пленок $\mathrm{CH}_{3} \mathrm{NH}_{3} \mathrm{PbBr}_{3}$ возбуждались с помощью ультрафиолетового светодиода LED UVTOP280TO39HS с длиной волны излучения $285 \mathrm{~nm}$. Излучение светодиода пропускалось через ультрафиолетовый фильтр UFS-8 и фокусировалось на поверхности образца при помощи кварцевой линзы с углом $\sim 10^{\circ}$ к нормали к поверхности образца в пятно диаметром $\sim 2 \mathrm{~mm}$. Спектры ФЛ регистрировались высокочувствительным волоконно-оптическим спектрометром со сверхмалым световым рассеянием „AVANTES“ - AvaSpec-ULSi2048L-USB2 OEM, paбoтающим в спектральном диапазоне $322-1100 \mathrm{~nm}$ со спектральным разрешением $4 \mathrm{~nm}$. ВАХ ПТ на основе
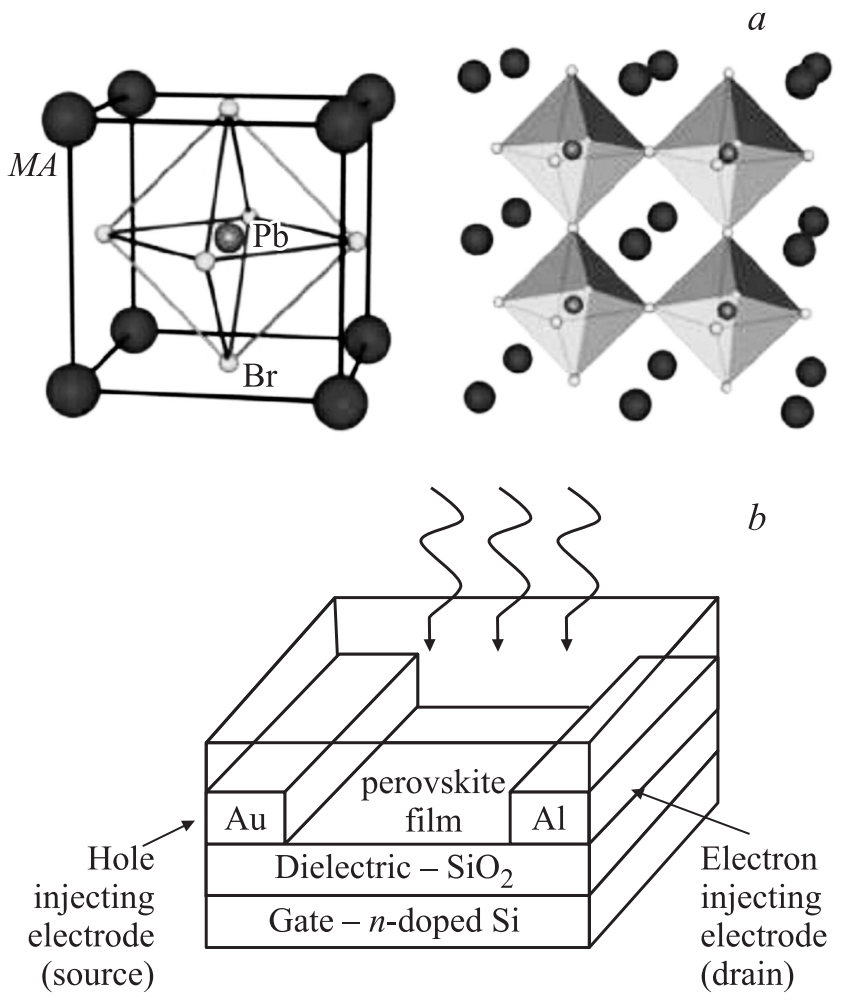

Рис. 1. Молекулярная структура $\mathrm{CH}_{3} \mathrm{NH}_{3} \mathrm{PbBr}_{3}(a)$, структура ПТ на основе пленки $\mathrm{CH}_{3} \mathrm{NH}_{3} \mathrm{PbBr}_{3}(b)$.

$\mathrm{CH}_{3} \mathrm{NH}_{3} \mathrm{PbBr}_{3}$ измерялись в вакууме $\left(3 \cdot 10^{-3}\right.$ Torr $)$ в темноте при $300 \mathrm{~K}$ на держателе азотного криостата с использованием автоматизированной измерительной установки на основе пикоамперметра Keithley 6487 и программируемого источника питания AKIP-1124. Напряжения на стоке-истоке и на затворе варьировались с переменным шагом в пределах от -18 до $+18 \mathrm{~V}$. Электрические контакты к образцам изготовлялись с использованием серебряной проволоки, которая крепилась к металлическим электродам серебряной пастой. Полевая подвижность носителей заряда $\mu_{\mathrm{FET}}$ оценивалась из ВАХ ПТ на основе пленок $\mathrm{CH}_{3} \mathrm{NH}_{3} \mathrm{PbBr}_{3}$ в режимах насыщения и слабых полей с помощью соотношения [14]:

$$
\begin{gathered}
I_{\mathrm{SD}}=(W / 2 L) \mu_{\mathrm{FET}} C_{\mathrm{I}}\left(V_{\mathrm{G}}-V_{\mathrm{th}}\right)^{2}, \\
I_{\mathrm{SD}}=(W / L) \mu_{\mathrm{FET}} C_{\mathrm{I}}\left(V_{\mathrm{G}}-V_{\mathrm{th}}\right) V_{\mathrm{SD}},
\end{gathered}
$$

где $W$ и $L-$ ширина и длина канала соответственно, $C_{\mathrm{I}}$ - емкость на единицу площади $\mathrm{SiO}_{2}$ (для толщины $\left.\sim 200 \mathrm{~nm}, \mathrm{C}_{\mathrm{I}} 10 \mathrm{nF} / \mathrm{cm}^{2}\right), V_{\mathrm{G}}$ - напряжение на затворе, $V_{\text {th }}$ - пороговое напряжение, соответствующее началу аккумуляционного режима. Величина $V_{\text {th }}$ определялась из передаточных характеристик - наклона зависимости $I_{\mathrm{SD}}^{0.5}$ от $V_{\mathrm{G}}$. 


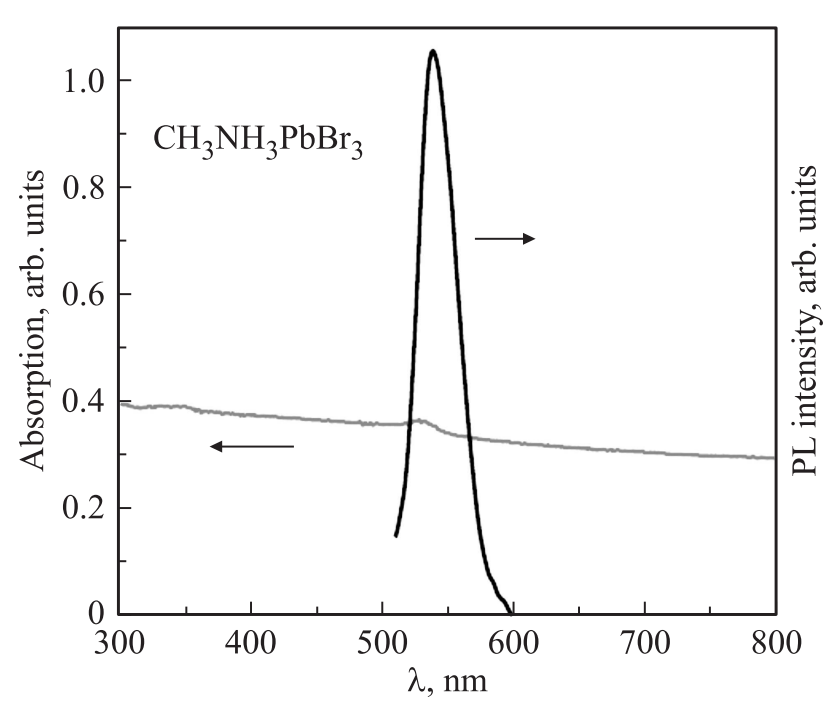

Pис. 2. Спектры поглощения и ФЛ-пленок $\mathrm{CH}_{3} \mathrm{NH}_{3} \mathrm{PbBr}_{3}$.

\section{3. Результаты и обсуждение}

На рис. 2 приведены спектры поглощения и ФЛ-пленок $\mathrm{CH}_{3} \mathrm{NH}_{3} \mathrm{PbBr}_{3}$, измеренные при $300 \mathrm{~K}$. Как видно из рис. 2, спектр поглощения пленки $\mathrm{CH}_{3} \mathrm{NH}_{3} \mathrm{PbBr}_{3}$ имеет край поглощения в спектральном интервале $\sim 530-540 \mathrm{~nm}$, что хорошо согласуется с шириной запрещенной зоны $E_{g} \sim 2.3 \mathrm{eV}$ исследуемого образца $\mathrm{CH}_{3} \mathrm{NH}_{3} \mathrm{PbBr}_{3}$. Спектр ФЛ того же образца лежит в спектральной области $\sim 500-600 \mathrm{~nm}$ и его максимум хорошо согласуется с положением края поглощения. На рис. 3 показаны результаты АCM исследований пленок $\mathrm{CH}_{3} \mathrm{NH}_{3} \mathrm{PbBr}_{3}$ вне рабочего канала ПТ, нанесенных на подложки методом полива при $\sim 100^{\circ} \mathrm{C}$. Как следует из анализа результатов АСМ, морфология пленок $\mathrm{CH}_{3} \mathrm{NH}_{3} \mathrm{PbBr}_{3}$ является довольно неоднородной и характеризуется наличием зерен с выраженными границами, при этом средний диаметр зерен составил $\sim 800 \mathrm{~nm}$, а их высота $\sim 100 \mathrm{~nm}$ (рис. 3). Исследованные пленки $\mathrm{CH}_{3} \mathrm{NH}_{3} \mathrm{PbBr}_{3}$ обладали значительной шероховатостью (Root Mean Square, $\mathrm{Rq} \sim 23 \mathrm{~nm}$ ). На рис. 4 показаны типичные BAX ПТ на основе пленки $\mathrm{CH}_{3} \mathrm{NH}_{3} \mathrm{PbBr}_{3}$ c $\mathrm{Au}-\mathrm{Al}$ сток-исток электродами, измеренные в вакууме, в темноте и при освещении с помощью имитатора солнечного света, при различных отрицательных напряжениях на затворе, $V_{\mathrm{G}}$. Как видно из рис. 4 , BAX ПТ на основе $\mathrm{CH}_{3} \mathrm{NH}_{3} \mathrm{PbBr}_{3}$ свидетельствуют о дырочном транспорте и характеризуются хорошо выраженными линейным режимом и режимом насыщения. Важно отметить, что ПТ на основе $\mathrm{CH}_{3} \mathrm{NH}_{3} \mathrm{PbBr}_{3}$ работают при низких напряжениях на стоке-истоке $\left(V_{\mathrm{SD}}\right)$ и на затворе, $V_{\mathrm{G}}$ (менее $15 \mathrm{~V}$ ). На рис. 5, $a$ показаны передаточные характеристики и их гистерезис для того же ПТ на основе $\mathrm{CH}_{3} \mathrm{NH}_{3} \mathrm{PbBr}_{3}$ при отрицательных и положительных напряжениях $V_{\mathrm{G}}$ в диапазоне от $-18 \mathrm{~V}$ до $+18 \mathrm{~V}$ при $V_{\mathrm{SD}}=-2 \mathrm{~V}$ и $-4 \mathrm{~V}$, измеренные в темноте. Как следует из рис. $5, a$, передаточные характеристики, измеренные при $V_{\mathrm{SD}}=-2 \mathrm{~V}$ и $-4 \mathrm{~V}$, почти аналогичны, таким образом они слабо зависят от $V_{\mathrm{SD}}$. Как видно из pис. $5, a, b$, ПТ на основе $\mathrm{CH}_{3} \mathrm{NH}_{3} \mathrm{PbBr}_{3}$ может работать
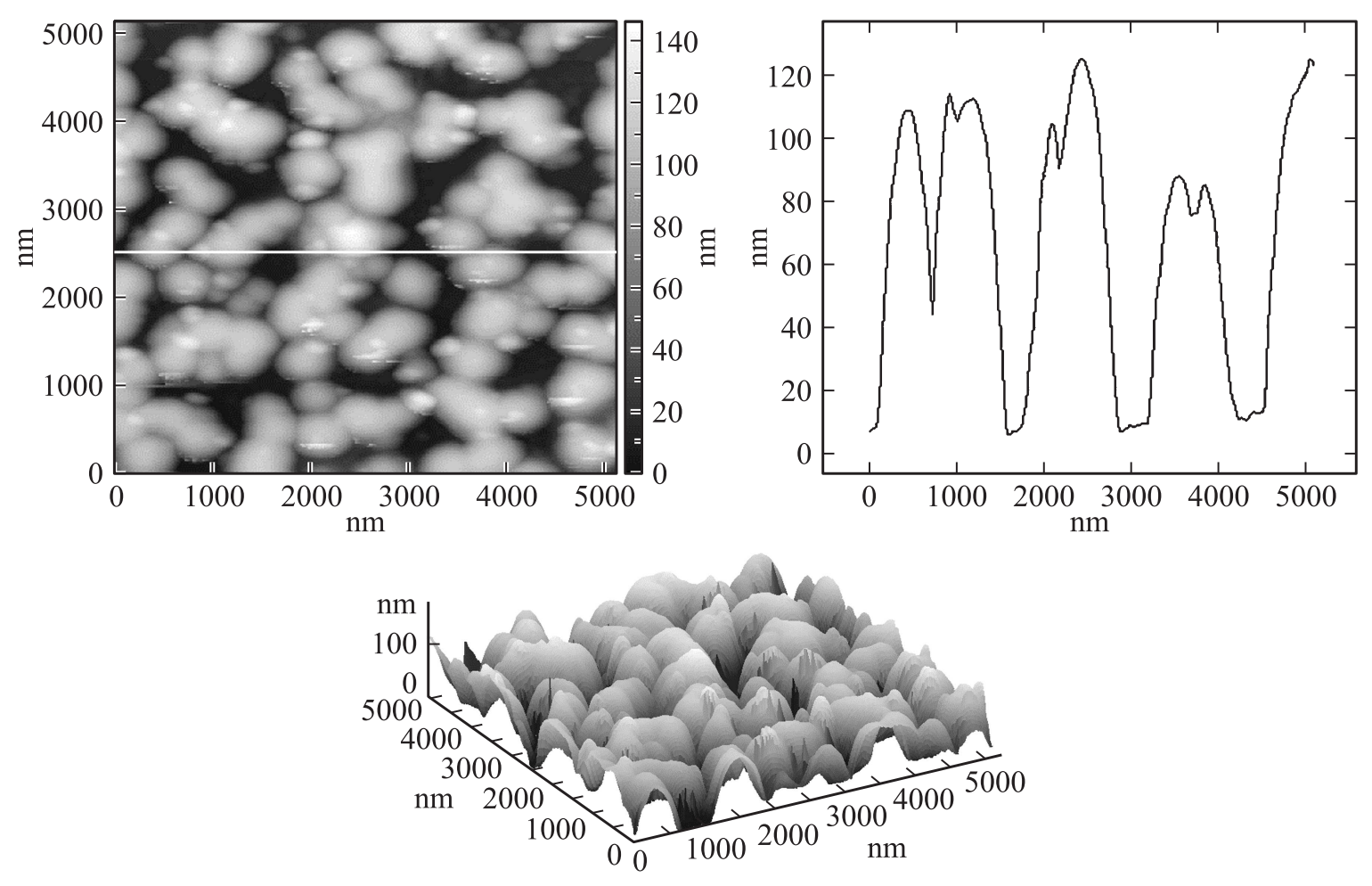

Рис. 3. Результаты АCM-исследований пленки $\mathrm{CH}_{3} \mathrm{NH}_{3} \mathrm{PbBr}_{3}$ вне канала ПТ. 


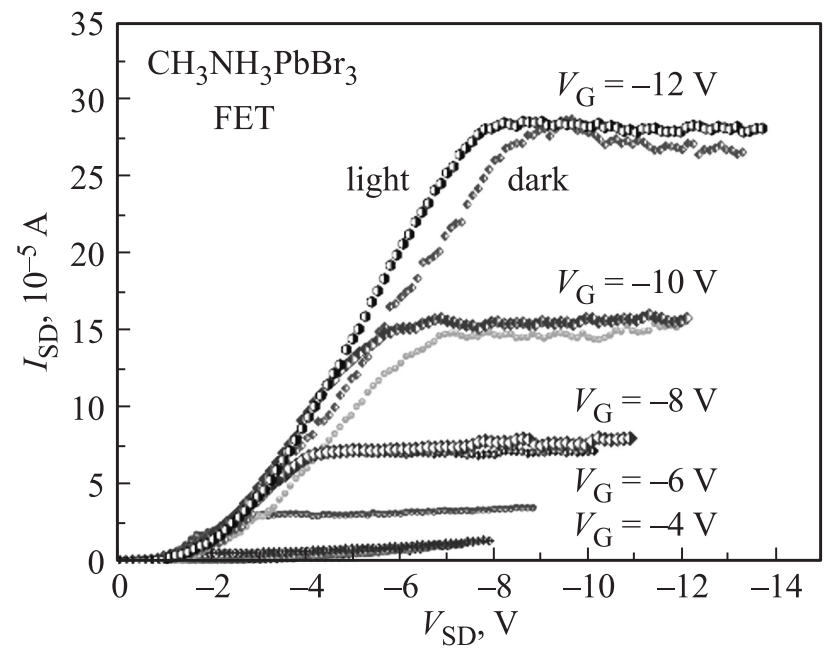

Pис. 4. $\mathrm{BAX}$ ПТ на основе $\mathrm{CH}_{3} \mathrm{NH}_{3} \mathrm{PbBr}_{3}$ для отрицательных значений $V_{\mathrm{G}}$, измеренные в темноте и при освещении имитатором солнечного света.

как в режиме накопления дырок, так и электронов, т.е. демонстрирует амбиполярное поведение в темноте и при освещении. Важно отметить небольшой гистерезис передаточных характеристик, наблюдаемый в ПТ на основе $\mathrm{CH}_{3} \mathrm{NH}_{3} \mathrm{PbBr}_{3}$, который незначительно возрастает с ростом $V_{\mathrm{SD}}$ (рис. 5,a). Наблюдаемый в наших ПТ структурах гистерезис передаточных характеристик значительно меньше, чем гистерезис, наблюдавшийся в ПТ на основе $\mathrm{CH}_{3} \mathrm{NH}_{3} \mathrm{PbI}_{3}$ в работе [13], что может означать относительно невысокую плотность ловушек в наших ПТ структурах. Отношение on/off, которое характеризует отношение тока через ПТ при подаче смещения на затвор к току без смещения в исследованных ПТ, достигает $\sim 10^{4}$ как при положительных, так и при отрицательных значениях $V_{\mathrm{G}}$ в интервале от $-18 \mathrm{~V}$ до $+18 \mathrm{~V}$ (рис. 5, $b$ ). Подвижности дырок при $300 \mathrm{~K}$, $\mu_{\text {hole }}(300 \mathrm{~K})$, в ПТ на основе $\mathrm{CH}_{3} \mathrm{NH}_{3} \mathrm{PbBr}_{3}$ рассчитывались с использованием формул (1) и (2) для режимов насыщения и слабых полей соответственно. Для ПТ на основе $\mathrm{CH}_{3} \mathrm{NH}_{3} \mathrm{PbBr}_{3}$, представленного на рис. 4,5 , пороговые напряжения, $V_{\mathrm{th}}$, оценивались из наклона зависимостей $I_{\mathrm{SD}}^{0.5}$ от $V_{\mathrm{G}}$ при $V_{\mathrm{SD}}=-2 \mathrm{~V}$ (см. вставку к рис. $5, b)$ и составили $\sim-3.6 \mathrm{~V}$ и $-0.8 \mathrm{~V}$ для отрицательных и положительных $V_{\mathrm{G}}$ соответственно. Значения $\mu_{\text {hole }}$ $(300 \mathrm{~K})$, оцененные при $300 \mathrm{~K}$ из ВАХ ПТ на основе $\mathrm{CH}_{3} \mathrm{NH}_{3} \mathrm{PbBr}_{3}$, составили $\sim 5 \mathrm{~cm}^{2} / \mathrm{Vs}$ (при $V_{\mathrm{G}}=-10 \mathrm{~V}$ и $V_{\mathrm{SD}}=-8 \mathrm{~V}$ ) и $\sim 2 \mathrm{~cm}^{2} / \mathrm{Vs}$ (при $V_{\mathrm{G}}=-10 \mathrm{~V}$ ) для режимов насыщения и слабых полей соответственно. Подвижность электронов при $300 \mathrm{~K}$ для ПТ на основе $\mathrm{CH}_{3} \mathrm{NH}_{3} \mathrm{PbBr}_{3}$ в режиме слабых полей, $\mu_{\text {elec }}(300 \mathrm{~K})$ составила $\sim 3 \mathrm{~cm}^{2} / \mathrm{Vs}$ (при $V_{\mathrm{G}}=1-2 \mathrm{~V}$ и $V_{\mathrm{SD}}=-2 \mathrm{~V}$ ). Близкие значения подвижности были получены и для ряда других ПТ на основе $\mathrm{CH}_{3} \mathrm{NH}_{3} \mathrm{PbBr}_{3}$ с токами отключения порядка $\sim 8 \cdot 10^{-8}-10^{-7} \mathrm{~A}$. Отношения on /off, полученные из передаточных характеристик, показанных на рис. $5, b$, составляли $\sim 10^{3}-10^{4}$ при отрицательных и положительных $V_{\mathrm{G}}(-18 \mathrm{~V}$ и $+18 \mathrm{~V})$, что по порядку величины близко к значениям on/off, полученным для органических ПТ на основе композитов полимернаночастицы $\mathrm{ZnO}[15,16]$. При освещении ПТ на основе $\mathrm{CH}_{3} \mathrm{NH}_{3} \mathrm{PbBr}_{3}$ с помощью имитатора солнечного света наблюдалось слабое увеличение $I_{\mathrm{SD}}(8-10 \%$ при $\left.V_{\mathrm{SD}}=-10 \mathrm{~V}\right)$ и небольшой сдвиг порогового напряжения $V_{\text {th }}$ (от $\sim-3.6$ и $\sim-0.8 \mathrm{~V}$ в темноте к $\sim 3.4 \mathrm{~V}$ и $\sim 1.4 \mathrm{~V}$ при освещении для отрицательных и положительных $V_{\mathrm{G}}$ соответственно). Слабая светочувствительность ПТ на основе $\mathrm{CH}_{3} \mathrm{NH}_{3} \mathrm{PbBr}_{3}$ может быть связана с особенностями поглощения света в композитных (органика-неорганика) полупроводниках, где возбуждение электронов светом сопровождается образованием экситонов. Диссоциация экситонов на границе раздела двух полупроводников (донора и акцептора) приводит к генерации свободных носителей заряда, транспорт которых осуществляется на донорно-акцепторном интер-
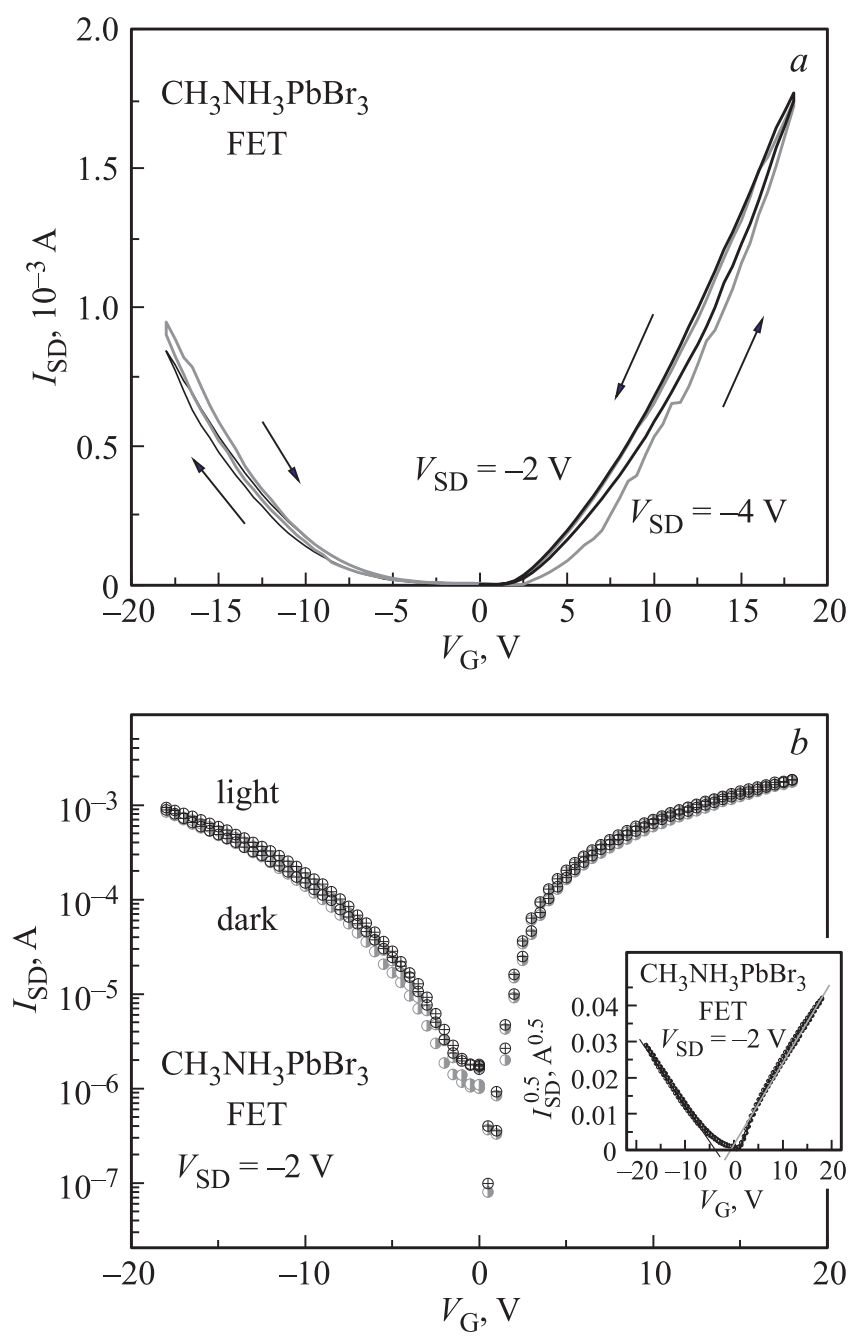

Рис. 5. Гистерезис передаточных характеристик ПТ на основе $\mathrm{CH}_{3} \mathrm{NH}_{3} \mathrm{PbBr}_{3}$ при $V_{\mathrm{SD}}=-2 \mathrm{~V}$ и $-4 \mathrm{~V}(a)$, передаточные характеристики ПТ на основе $\mathrm{CH}_{3} \mathrm{NH}_{3} \mathrm{PbBr}_{3}$ при $V_{\mathrm{SD}}=-2 \mathrm{~V}$, измеренные в темноте и при освещении имитатором солнечного света $(b)$. Зависимость $I^{0.5}$ от $V_{\mathrm{G}}$ при $V_{\mathrm{SD}}=-2 \mathrm{~V}$ для того же ПТ (вставка). 
фейсе. Диффузионная длина экситонов в органических полупроводниках ограничена расстоянием $~ 10 \mathrm{~nm}$ из-за их низкой подвижности [17], в то время как в перовскитах, таких как $\mathrm{CH}_{3} \mathrm{NH}_{3} \mathrm{PbI}_{3}$, диффузионная длина экситонов достигает $\sim 100-120 \mathrm{~nm}[18]$. Вклад в фототок в таких системах дают только фотоны, поглощенные на характерной длине диффузии экситона вблизи плоскости гетероперехода, которые могут эффективно двигаться к интерфейсу, обеспечивая генерацию носителей заряда. В активных слоях СЭ на основе металлорганических перовскитов толщиной несколько сотен $\mathrm{nm}$ вклад в фототок дают менее $10 \%$ поглощенных фотонов. В ПТ на основе $\mathrm{CH}_{3} \mathrm{NH}_{3} \mathrm{PbBr}_{3}$ расстояния между планарными электродами $\mathrm{Al}-\mathrm{Au}$ составляли $\sim 7 \mu \mathrm{m}$, что значительно превышает длины диффузии экситонов $\sim 100 \mathrm{~nm}$ и $\sim 360 \mathrm{~nm} \mathrm{в} \mathrm{CH}_{3} \mathrm{NH}_{3} \mathrm{PbBr}_{3}$ в темноте и при освещении соответственно [19]. В связи с этим большинство фотогенерированных экситонов в таких ПТ-структурах диссоциируют вдали от электродов, не внося вклад в фототок, что приводит к низкой фоточувствительности, наблюдаемой нами в эксперименте.

Полученные нами ПТ на основе $\mathrm{CH}_{3} \mathrm{NH}_{3} \mathrm{PbBr}_{3}$ демонстрируют при $300 \mathrm{~K}$ работу в режимах насыщения и слабых полей, при этом значения дырочной подвижности превышают величины подвижности $\sim 1 \mathrm{~cm}^{2} / \mathrm{Vs}$ в ПТ на основе $\mathrm{CH}_{3} \mathrm{NH}_{3} \mathrm{PbI}_{3-x} \mathrm{Cl}_{x}$ [5], а также значения подвижности дырок $\sim 4.7 \mathrm{~cm}^{2} / \mathrm{Vs}$, полученные для органических ПТ на основе полимерных композитов P3HT : [70]PCBM : Ni [20]. По аналогии с результатами работы [13] можно полагать, что улучшение характеристик ПТ на основе $\mathrm{CH}_{3} \mathrm{NH}_{3} \mathrm{PbBr}_{3}$, по сравнению с ПТ на основе $\mathrm{CH}_{3} \mathrm{NH}_{3} \mathrm{PbI}_{3-x} \mathrm{Cl}_{x}$ [5], может быть связано со снижением концентрации точечных дефектов, расположенных на границах зерен. При этом, согласно модели, предложенной в работе [13], транспорт носителей заряда в исследуемых структурах при $300 \mathrm{~K}$ ограничен процессом миграции ионов при наличии точечных дефектов на границах зерен. Другим возможным механизмом, затрудняющим транспорт в ПТ-структурах на основе металлорганических перовскитов, является поляризационная разупорядоченность дипольных моментов $\mathrm{MA}^{+}$ катионов при $300 \mathrm{~K}$ [13]. Можно полагать, что, по аналогии со свойствами ПТ на основе $\mathrm{CH}_{3} \mathrm{NH}_{3} \mathrm{PbI}_{3}[7,13]$, эти механизмы транспорта должны вымораживаться при понижении температуры, при этом возможно еще большее увеличение подвижности в ПТ на основе $\mathrm{CH}_{3} \mathrm{NH}_{3} \mathrm{PbBr}_{3}$.

\section{4. Заключение}

Разработаны и исследованы электрические и оптические свойства ПТ-структур на основе растворимых металлорганических перовскитов $\mathrm{CH}_{3} \mathrm{NH}_{3} \mathrm{PbBr}_{3}$. Показано, что такие структуры демонстрируют ВАХ, характерные для амбиполярных ПТ с режимом насыщения. Передаточные характеристики ПТ на основе $\mathrm{CH}_{3} \mathrm{NH}_{3} \mathrm{PbBr}_{3}$ обладают незначительным гистерезисом и слабо зависят от напряжения на стоке--истоке. Значения $\mu_{\text {hole }}(300 \mathrm{~K})$, рассчитанные из ВАХ ПТ на основе
$\mathrm{CH}_{3} \mathrm{NH}_{3} \mathrm{PbBr}_{3}$ в режимах насыщения и слабых полей, составляют $\sim 5 \mathrm{~cm}^{2} / \mathrm{Vs}$ и $\sim 2 \mathrm{~cm}^{2} / \mathrm{Vs}$, а подвижность электронов $\mu_{\text {elec }}(300 \mathrm{~K}) \sim 3 \mathrm{~cm}^{2} / \mathrm{Vs}$, что превышает значение подвижности $\sim 1 \mathrm{~cm}^{2} / \mathrm{Vs}$, полученное ранее для полевых транзисторов на основе $\mathrm{CH}_{3} \mathrm{NH}_{3} \mathrm{PbI}_{3}$. Предположено, что транспорт носителей заряда в ПТ структурах на основе металлорганических перовскитов при $300 \mathrm{~K}$ ограничен процессами миграции ионов при наличии точечных дефектов, связанных с границами зерен, а также поляризационной разупорядоченностью дипольных моментов $\mathrm{MA}^{+}$катионов.

\section{Список литературы}

[1] National Renewable Energy Laboratory, Best Research Cell Efficiencies, www.nrel.gov/ncpv/images/efficiency_chart.jpg; accessed: June 2016.

[2] N.J. Jeon, J.H. Noh, W.S. Yang, Y.C. Kim, S. Ryu, J. Seo, S.I. Seok. Nature 517, 476 (2015).

[3] H. Zhou, Q. Chen, G. Li, S. Luo, T.-B. Song, H.-S. Duan, Z. Hong, J. You, Y. Liu, Y. Yang. Science 345, 542 (2014).

[4] H.S. Jung, N.-G. Park. Small 11, 10 (2015).

[5] V. Mei, C. Zhang, Z.V. Vardeny, O.D. Jurchescu. MRS Commun. 5, 297 (2015).

[6] J.H. Heo, S.H. Im, J.H. Noh, T.N. Mandal, C.-S. Lim, J.A. Chang, Y.H. Lee, H. Kim, A. Sarkar, M.K. Nazeeruddin, M. Gretzel, S.I. Seok. Nature Photonics 7, 486 (2013).

[7] X.Y. Chin, D. Cortecchia, J. Yin, A. Bruno, C. Soci. Nature Commun. 6, 7383 (2015).

[8] J.G. Labram, D.H. Fabini, E.E. Perry, A.J. Lehner, H. Wang, A.M. Glaudell, G. Wu, H. Evans, D. Buck, R. Cotta, L. Echegoyen, F. Wudl, R. Seshadri, M.L. Chabinyc. J. Phys. Chem. Lett. 6, 3565 (2015).

[9] G. Wang, D. Li, H.-C. Cheng, Y. Li, C.-Y. Chen, A. Yin, Z. Zhao, Z. Lin, H. Wu, Q. He, M. Ding, Y. Liu, Y. Huang, X. Duan. Sci. Adv. 1, e1500613 (2015).

[10] C.R. Kagan, D.B. Mitzi, C.D. Dimitrakopoulos. Science 286, 945 (1999).

[11] T. Matsushima, K. Fujita, T. Tsutsui. Jpn. J. Appl. Phys. 43, L1199 (2004).

[12] T. Matsushima, S. Hwang, A.S.D. Sandanayaka, C. Qin, S. Terakawa, T. Fujihara, M. Yahiro, C. Adachi. Adv. Mater. 28, 10275 (2016).

[13] S.P. Senanayak, B. Yang, T.H. Thomas, N. Giesbrecht, W. Huang, E. Gann, B. Nair, K. Goedel, S. Guha, X. Moya, C.R. McNeill, P. Docampo, A. Sadhanala, R.H. Friend, H. Sirringhaus. Sci. Adv. 3, e1601935 (2017).

[14] C.D. Dimitrakopoulos, P.R.L. Malenfant. Adv. Mater. 14, 99 (2002).

[15] A.N. Aleshin, I.P. Shcherbakov, V.N. Petrov, A.N. Titkov. Organic Electron. 12, 1285 (2011).

[16] А.Н. Алешин. УФН, 183, 657 (2013).

[17] D.E. Markov, J.C. Hummelen, P.W.M. Blom, A.B. Sieval. Phys. Rev. B 72, 045216 (2005).

[18] S.D. Stranks, G.E. Eperon, G. Grancini, C. Menelaou, M.J.P. Alcocer, T. Leijtens, L.M. Herz, A. Petrozza, H.J. Snaith. Science 342, 341 (2013).

[19] N. Kedem, T.M. Brenner, M. Kulbak, N. Schaefer, S. Levcenko, I. Levine1, D. Abou-Ras, G. Hodes, D. Cahen. J. Phys. Chem. Lett. 6, 2469 (2015).

[20] A.N. Aleshin, I.P. Shcherbakov, I.N. Trapeznikova, V.N. Petrov. Phys. Solidi. State 58, 1882 (2016). 